\title{
CORRIGENDUM
}

doi:10.1038/nature09412

\section{MicroRNA-mediated integration of haemodynamics and Vegf signalling during angiogenesis}

Stefania Nicoli, Clive Standley, Paul Walker, Adam Hurlstone, Kevin E. Fogarty \& Nathan D. Lawson

\section{Nature 464, 1196-1120 (2010)}

It has been brought to our attention that the panels in Figs $3 g$ (control MO, left panel) and $4 \mathrm{i}$ (uninjected) are the same image. During the preparation of our manuscript for submission, this panel was inadvertently duplicated and used in both figures. It was also labelled incorrectly in Fig. 3g. The correct Fig. 3g control panel (scrambled MO-injected embryo) and the original experimental panel ( $m i R-126$ MO-injected embryo) are shown here. As shown in several other figures of our manuscript (for example, Figs 1j, 2b and 4k), embryos injected with control MO show normal AA5x formation, similar to uninjected embryos. This mistake does therefore not affect the interpretation of or conclusions made from our results. We apologize for any confusion this error may have caused.

Control MO
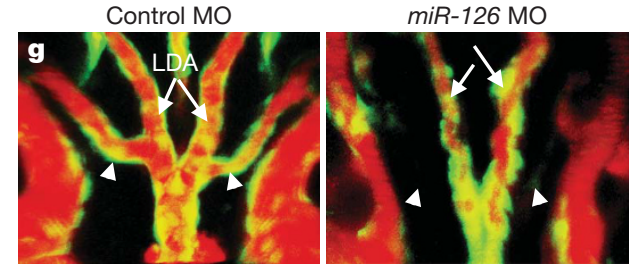